\title{
Superior Mesenteric Artery Syndrome: A Worldwide Descriptive Study with Literature Review
}

Ruhidayati Awaludin, ${ }^{1}$ Hazimah Ab Rahim,, ${ }^{1}$ Dg. Syazana Arivai, ${ }^{1}$ Mostafa Refaie Elkeleny. ${ }^{1}$

\begin{abstract}
Background: Superior mesenteric artery syndrome is best described as compression of the third part of duodenum by the superior mesenteric artery, resulting in obstruction. This rare condition has been studied for decades yet remains obscure. This study aimed to analyze different clinical presentations, diagnostic modalities, treatment approaches and outcomes of this condition. Methods: Thirty-five superior mesenteric artery syndrome cases were collected retrospectively from a Facebook group called "Superior Mesenteric Artery Syndrome Awareness at Support". A questionnaire was designed using Coogle Forms to obtain the demographics, presenting symptoms, risk factors and co-morbidities, investigations, means of treatment and the outcomes. Data was entered into Microsoft office Excel for statistical analysis. Results: The median age at diagnosis was 22 years. The median body mass index was $20.8 \mathrm{~kg} / \mathrm{m} 2$. The median time interval from symptom onset to initial diagnosis was 22 months. The major presenting symptoms were abdominal pain $(82.9 \%)$, nausea $(77.1 \%)$, and vomiting $(65.7 \%)$. Abdominal computed tomography scan with contrast (82.9\%) was commonly used for confirmation of diagnosis. Thirteen cases $(37.1 \%)$ were congenital. Thirty patients $(85.7 \%$ ) had received treatment. The overall management success was only $13.3 \%$. Surgical management (34.3\%) was the most commonly used regimen. Conclusion: Diagnosis of superior mesenteric artery syndrome is established after a thorough assessment of the clinical presentations and confirmed with suitable imaging modalities. The choice of treatment should be dependent on the causes and severity as different patients respond differently to therapy. Recurrence is possible in all patients, and a long-term follow up is thus required.
\end{abstract}

Keywords: Superior Mesenteric Artery Syndrome; Duodenal Obstruction (Source: MeSH-NLM).

About the Author: Ruhidayati Awaludin is a final-year medical student at the Faculty of Medicine, Alexandria University, Egypt.

\section{Introduction}

Superior mesenteric artery syndrome (SMAS) is a rare medical condition caused by compressive obstruction of the transverse or ascending portion of duodenum by the superior mesenteric artery (SMA) ${ }^{1-6}$ The obstruction results from the loss of the intervening mesenteric fat pad between the aorta and SMA, leading to narrowing of the angle between these two vesse1s. ${ }^{7.8}$ Duodenal compression by the SMA was first described as results of autopsy by Carl Freiherr von Rokitansky in 1861. 3,6,8-12 It has since been given other names such as aortomesenteric artery compression, arteriomesenteric artery duodenal compression, duodenal vascular compression, cast syndrome and Wilkie syndrome. ${ }^{17.9}$ Wilkie explicated the clinical pathology in 1927 , and since then more cases were reported. ${ }^{1,2,5,7,7,12,13}$

SMA arises from the abdominal aorta at the level of the first lumbar vertebra. ${ }^{2,4,9,14,15}$ It usually leaves the aorta at an angle of $25^{\circ}-60^{\circ}$, leaving a considerable space for the left renal vein, third portion of duodenum, and uncinate process of the pancreas to pass through. ${ }^{15,16}$ The distance between the SMA and the aorta normally ranges from $10-28 \mathrm{~mm}^{12,16}$ The presence of retroperitoneal fat plays an important role to keep the angle and distance within this normal range. Any factors which contribute to the loss of this fat such as rapid weight loss, increased lordosis and external pressure will risk an individual for the development of SMAS. $1,9,14,17$
SMAS is usually associated with common gastrointestinal obstruction symptoms such as abdominal pain, post-prandial abdominal pain, nausea, vomiting, weight loss or poor weight gain, slow digestion or indigestion, bloating, abdominal distention, feeling of fullness despite small amount of food intake, malaise and lethargy. The exact etiology of SMAS is not known, but certain predisposing conditions are clearly recognized, such as thin body build, rapid linear growth, severe injuries, dietary disorders especially anorexia nervosa, anatomic anomalies, central nervous system and spinal disease. ${ }^{3-5,7-7,9,11,14,18,19}$

The diagnosis of SMAS is frequently made by exclusion and relies on a high index of suspicion. 8,14,19 The symptoms of this disorder may resemble many other gastrointestinal problems thus necessitating further confirmation by imaging. ${ }^{8,18}$ Upper gastrointestinal (UGI) series was once considered as the confirmatory test of choice. ${ }^{5,8}$ The radiologic criteria includes: (1) dilatation of the first and second parts of the duodenum, (2) an abrupt vertical or oblique compression of the third duodenal portion, (3) retrograde flow of the contrast proximal to the compression, (4) delay of transit of the contrast into jejunum for at least 4-6 hours, and (5) a relief of the compression and symptoms in a knee-to-chest or left lateral decubitus position. ${ }^{9,20}$ Computed tomography (CT) scan or CT angiography (CTA) assess the aortomesenteric angle and distance as well as the extent of duodenal distension. ${ }^{3}$ In SMAS cases, the SMA

' Faculty of Medicine, Alexandria University, Alexandria, Egypt. 
angle to the aorta is decreased to $6^{\circ}-25^{\circ}$, whereas the distance is decreased to $2-8 \mathrm{~mm} .{ }^{16}$

Therapy for SMAS can be either conservative or surgical depending on the causes and severity. Conservative measures included weight gain, positioning, medications, enteral feeding, and parenteral feeding. Nasogastric decompression, correction of the fluid and electrolyte balance, and nutritional support are beneficial during an acute attack. ${ }^{79,21}$ Small, frequent high caloric diet with postprandial postural modifications such as knee-to-chest or right lateral decubitus and the use of prokinetic drugs such as metoclopramide are recommended. 7.9,18,20-22 Enteral feeding by placement of nasojejunal tube distal to the obstruction is needed when oral feeding is not well-tolerated. ${ }^{\text {t5 }}$ Total parenteral nutrition (TPN) can be an alternative.? In both practices, the patients should be monitored for refeeding syndrome as it might occur easily in malnourished patients. ${ }^{14}$

Surgical intervention is indicated when medical treatment fails. Other indications include long standing cases, profound dilatation and stasis of the proximal small bowel, preference of the patient for surgical correction rather than prolonged conservative management, and the presence of associated diseases such as peptic ulcer and pancreatitis. ${ }^{10,11,23}$ The obstruction is preferably bypassed with an anastomosis such as gastrojejunostomy or duodenojejunostomy. Detachment of the duodenum away from the narrowed aortomesenteric angle through a procedure called Strong's procedure might be helpful. Some modifications to this technique may enhance the outcome, and this procedure is known as duodenal de-rotation.

SMAS is a well-recognized clinical entity, yet the diagnosis is frequently delayed and the condition is often treated inappropriately due to the lack of awareness among physicians. It may resemble many other gastrointestinal problems, hence complicating diagnosis, and may be followed by rapid progression to a life-threatening state such as malnutrition, dehydration, electrolytes imbalance and even death. ${ }^{4,14,18} \mathrm{New}$ studies in various aspects are needed to ensure early recognition of the condition together with improvement in the management and outcomes. The objective of this study is to analyze different clinical presentations, diagnostic modalities, treatment approaches and outcomes of SMAS. The outcomes of this study may be helpful in conducting future studies.

\section{Methods}

Thirty-five SMAS cases were collected retrospectively from a Facebook group called "Superior Mesenteric Artery (SMA) Syndrome Awareness a Support" over a period of two months. The group was established in November 2009 and has assembled almost 2,000 members worldwide at present. It consists of SMAS patients, patients' friends, family and other members interested in this rare pathology.

A questionnaire was designed using Google Forms and distributed to the members on board. Strict rules were applied. Patients who were officially diagnosed were allowed to answer the questionnaire, regardless of treatment. The friends or family members were allowed to fill the form only if the patient was severely ill or disabled. The data collected included demographics, presenting symptoms, risk factors and co-morbidities, investigations, means of treatment, and the outcomes.

Demographic information collected included sex, age at time of initial diagnosis, height and weight. The weight status of each patient was estimated by calculation of the body mass index (BMI). Assessment of the BMI was made according to the following classification: underweight $\left(<18.5 \mathrm{~kg} / \mathrm{m}^{2}\right)$, normal $\left(18.5-24.9 \mathrm{~kg} / \mathrm{m}^{2}\right)$, overweight $\left(25.0-29.9 \mathrm{~kg} / \mathrm{m}^{2}\right)$, or obese $(330.0$ $\left.\mathrm{kg} / \mathrm{m}^{2}\right)$.

Both main and associated symptoms were recorded. Presenting symptoms were analyzed according to the duration before diagnosis. Diagnosis established within six months from the onset of symptoms was considered an early diagnosis, whereas those which took more than six months were considered to be late diagnosis. Possible predisposing factors for SMAS, presence of multiple co-morbidities, and surgical history for each case were explored.

The management for each case was grouped into: conservative, surgical, or both. The evaluation of treatment outcomes was made by the patients and classified into four grades: excellent (no symptoms after using the treatment), good (mild temporary symptoms without any need for therapy), satisfactory (moderate occasional symptoms that can be controlled by a particular body position or medications), and poor (moderate or severe symptoms essentially the same as those experienced preoperatively). All information was entered into Microsoft office Excel 2007 spreadsheet for statistical analysis.

\section{Results}

A total of 35 SMAS patients answered the questionnaire; 31 (88.6\%) of these were females. The median age at diagnosis was 22 years, with a range of 12-59 years. The median BMI was $20.8 \mathrm{~kg} / \mathrm{m}^{2}$, with a range of $14.2-26.4 \mathrm{~kg} / \mathrm{m}^{2}$. Six patients $(17.1 \%)$ were underweight, 26 patients $(74.3 \%)$ had a normal BMI, and three patients (8.6\%) were overweight.

The median time interval from symptom onset to initial diagnosis was 22 months, with a range of 3-216 months. Only five patients (14.3\%) were diagnosed early (Table 1). Most patients (94.3\%) presented with more than one symptom. The major presenting symptoms were abdominal pain (82.9\%), nausea (77.1\%), and vomiting $(65.7 \%)$. Other symptoms included weight loss $(48.6 \%)$, fatigue $(34.3 \%)$, bloating $(25.7 \%)$, feeling of fullness $(17.1 \%)$, altered bowel habit (17.1\%) and early satiety (11.4\%), as shown in Table 1.

Twenty-nine patients (82.9\%) underwent abdominal CT scan with contrast, 23 patients $(65.7 \%)$ underwent barium UGI series, 17 patients $(48.6 \%)$ underwent endoscopy, 13 patients $(37.1 \%)$ underwent ultrasonography, and 12 patients (34.3\%) underwent gastric emptying study.

Thirteen patients $(37.1 \%)$ had congenital SMAS, $11(31.4 \%)$ had pre-morbid conditions, while the remaining 11 patients $(31.4 \%)$ were free of co-morbidities. The most common co-morbid condition was sudden weight loss, followed by spinal problems, cerebral palsy, motor vehicle accident, history of anorexia, midgut mal-rotation, and iatrogenic (Table 1). About $60 \%$ of the cases were identified to have a previous surgical history prior to the diagnosis of SMAS. 
Table 1. Presenting Symptoms, Interval between Symptom Appearance and Diagnosis, and Pre-morbid Conditions of Survey Respondents

\begin{tabular}{ll}
\hline Patient condition & $\mathrm{n}(\%)$ \\
Symptom* & $29(82.9)$ \\
Abdominal pain & $27(77.1)$ \\
Nausea & $23(65.7)$ \\
Vomiting & $17(48.6)$ \\
Weight loss & $12(34.3)$ \\
Fatigue & $9(25.7)$ \\
Bloating & $6(17.1)$ \\
Feeling of fullness & $6(17.1)$ \\
Altered bowel habits & $4(11.4)$ \\
Early satiety & \\
Time Interval & $5(14.3)$ \\
0.5 year & $4(11.4)$ \\
0.5-1 year & $19(54.3)$ \\
1-5 years & $2(5.7)$ \\
5-10 years & $5(14.3)$ \\
$\geq 10$ years & \\
\hline Pre-morbid Condition & $13(37.1)$ \\
Congenital & $11(31.4)$ \\
Unknown & $5(14.3)$ \\
Sudden weight loss & $2(5.7)$ \\
Spinal problems & $1(2.9)$ \\
Cerebral palsy & $1(2.9)$ \\
Motor vehicle accident & $1(2.9)$ \\
History of anorexia & $1(2.9)$ \\
\hline latrogenic & \\
\hline
\end{tabular}

* Some patients presented with more than one symptom.

Conservative management was attempted in 18 patients (51.4\%). Nine of them tried weight gain by consuming high caloric diets, three were given medications (antacids and anti-emetics), two practiced the knee-to-chest position after meals, 11 used a feeding tube, and six had total TPN. Seven patients (38.9\%) did not respond well to the regimens and were further treated surgically. A total of 19 patients (54.3\%) underwent surgery. Duodenojejunostomy was performed on 15 patients ( $79.0 \%$ ), and two patients $(10.5 \%)$ were treated with gastrojejunostomy. Other procedures included Strong's procedure with duodenal de-rotation, SMA release, and untwisting of the malrotated duodenum (Table 2).

\section{Discussion}

In our study, we describe 35 SMAS patients who answered our questionnaire over a period of two months. Our data showed that females were affected more frequently than males, and the majority of patients were between 20-29 years of age. This finding is congruent with earlier studies, which reported the involvement of more than $64 \%$ female patients. ${ }^{57,13,1,19,21}$ Biank (2006) suggested that low BMI is not a prerequisite for the development of SMAS, which is in accordance with our study finding that $74.3 \%$ had normal BMI. However, the relationship between normal BMI and the occurrence of SMAS remains unclear. ${ }^{5}$

Several etiological factors contributing to SMAS have been reported worldwide. Congenital anomalies, including a possible genetic predisposition such as high attachment of ligament of Treitz and hypertrophy of the ligament, midgut mal-rotation as found in one of our cases, and visceral ptosis may share their roles in compression of the duodenum. 2,9,10,13,15 Visceral ptosis allows dragging of small intestines into the true pelvis without resting on the pelvic floor, causing excessive traction of the SMA hence duodenal compression. ${ }^{15}$

Another cause is depletion of mesenteric fat which occurs following severe and rapid weight loss, from debilitating injuries, during catabolic states in excessive burn, and intravenous drug addictions (cocaine and heroin) with daily minimal food intake. ${ }^{10,15,18}$ Among the patients who answered our survey, $14.3 \%$ had SMAS with sudden weight loss resulting from head injury, following brain surgery, hyperthyroidism, and psychological stress following Helicobacter pylori infection.

Compression from external devices (such as tight belts, wearing body spica cast for long time periods in vertebrae treatment) and spinal disease (such as increased lordosis) may cause traction of the SMA. $1,2,9,10,15$ One of our cases experienced external compression from outside the body resulting from a car accident, while two other patients had spinal lordosis following fall from an elevated height and scoliosis, respectively.

Disorders of the central nervous system may play a role in the occurrence of SMAS as we reviewed one case with cerebral palsy. Disabilities such as spastic quadriparesis, prolonged supine positioning, immobilization in spinal orthosis, and lumbar lordosis occurring in patient with cerebral palsy may induce duodenal compression. ${ }^{4,7}$ Prior studies state that SMAS could occur with psychic disorders such as anorexia nervosa $a^{9,19}$ and dietary disorders such as malabsorption. ${ }^{9}$ We recorded one case of anorexia nervosa with SMAS.

Table 2. Subjective Patient Perspective on Management and Outcomes

\begin{tabular}{|c|c|c|c|c|c|}
\hline \multirow{2}{*}{ Management (n) } & \multicolumn{5}{|c|}{ Outcome, n (\%) } \\
\hline & Excellent & Good & Satisfactory & Poor & Unclassified \\
\hline Not treated (5) & NA & NA & NA & NA & NA \\
\hline Conservative (11) & $2(18.2)$ & $4(36.4)$ & $2(18.2)$ & $3(27.3)$ & $0(0.0)$ \\
\hline Surgical (12) & $1(8.3)$ & $2(16.7)$ & $3(25.0)$ & $5(41.7)$ & $1(8.3)$ \\
\hline Both (7) & $1(14.3)$ & $0(0.0)$ & $3(42.9)$ & $3(42.9)$ & $0(0.0)$ \\
\hline
\end{tabular}

$\mathrm{NA}=$ not applicable. 
One female patient who was involved in our study developed SMAS after her deterioration of health following a partial hysterectomy with the $d a$ Vinci robot in February 2013. This advanced technique was believed to cause damage and severe organ prolapse which led to traction of SMA with the resultant duodenal compression. However, more evidence is needed to support this hypothesis.

Another factor that can be considered is the rapid growth in height without the appropriate and corresponding weight gain in children, which in turn narrows the aortomesenteric angle and causes SMAS. ${ }^{1,18}$ In addition, postoperative weight loss, prolonged bed rest as a result of chronic illness or severe trauma, asthenic habitus, sagittal kyphosis and scoliosis surgery had also been reported by some authors as contributing factors. ${ }^{1,4,17,19}$

Some important and typical complaints from patients are chronic post-prandial abdominal pain with intermittent exacerbation that are relieved by the knee-to-chest position, nausea, and progressive vomiting that increased in intensity and frequency. ${ }^{1,4,7,14-16,20,26}$ Vomitus usually consisted of undigested food.? This constellation of symptoms is concurrent with the majority of our cases.

Only five cases ( $14.3 \%$ ) were diagnosed as early as three months after the onset of symptoms. Most patients were diagnosed within 1-5 years of suffering, suggesting the lack of awareness among physicians regarding this rare disorder. Interestingly, it took almost 10 years or more for five $(14.3 \%)$ of our cases to be diagnosed. Delay in diagnosis and proper management will leads to the development of co-morbidities such as malnutrition, dehydration, electrolytes imbalance and even death. ${ }^{14}$

UGI series is usually sufficient for diagnosis in most cases. The appearance of dilated proximal duodenum with an abrupt discontinuation of the barium flow in the third part might also be seen in cases of megaduodenum. $9.15 \mathrm{UGl}$ series does not seem to be sensitive enough due to the intermittent course of SMAS symptoms, hence it is generally suitable during an active attack. ${ }^{8}$ Therefore, diagnosis of most of our patients $(82.9 \%)$ were established by contrast-enhanced abdominal CT scan, which could show the compression and reduced angle both in symptomatic and asymptomatic patients. Other signs of obstruction can also be seen such as a distended stomach and a dilated proximal duodenum. ${ }^{6,8,16}$

With regard to treatment, conservative measures should be attempted prior to surgery. ${ }^{10,11,24}$ The goal for this approach is to encourage restoration of retroperitoneal fat, with subsequent increase of the aortomesenteric angle and reduction in duodenal compression. ${ }^{18,14}$ Acute cases with a short history, moderate symptoms and incomplete duodenal obstruction may benefit from this regimen. ${ }^{11}$ In our review, the success rate of medical therapy is as low as 11.1, similar to that reported in the study by $\mathrm{Ha}$ et al. (14\%). ${ }^{13}$ Burrington (1976) ${ }^{9}$ reported that $70 \%$ of patients required surgical intervention in spite of the benefit in advances of both enteral and parenteral nutrition made in the last 30 years. Children and adults with a short history may benefit from this approach. However, most of these patients are critically ill, requiring prolonged hospital stay with a low

\section{success rate. ${ }^{19}$}

Gastrojejunostomy is considered when both the stomach and duodenum are severely dilated or in the presence of a duodenal ulcer. ${ }^{1,9,13,14}$ Difficulty in mobilizing the duodenum during a duodenojejunostomy or other procedures although indicated, may also requires this measure. ${ }^{14}$ It provides adequate gastric decompression but fails to completely release duodenal obstruction resulting in recurrent symptoms requiring another procedure.5.19 Some well-known complications include dumping syndrome, blind loop syndrome, gastric bile reflux and marginal ulceration. ${ }^{1,15,20}$ Two (10.5\%) of our cases underwent gastrojejunostomy. One of the patients was doing well after the surgery with little weight gain. However, the symptoms reappeared after four months and was reported to be milder than before.

Duodenojejunostomy was performed on 15 patients (79.0\%). This method has been favored and the vast majority of results have been excellent, with Wilkie alone reported to have successfully performed it on 64 patients with SMAS. ${ }^{13,15}$ Advances in laparoscopic surgery have led to reports of laparoscopic duodenojejunostomy showing excellent outcomes which was pioneered by Gersin and Heniford in 1998. ${ }^{5}$ Surprisingly, only two $(13.3 \%)$ of the respondents were successfully treated by this approach in spite of the high success rate reported by most authors. Symptoms persist in $80 \%$ of them ( 12 of 15 patients). The lack of detailed assessment of patients' condition and the presence of SMAS complications pre-operatively may contribute to the failure of such procedure. One had just underwent laparoscopic duodenojejunostomy five months prior to the surgery, thus making the outcome assessment impossible.

As both approaches produce interruption to the bowel integrity, Strong's operation has been advocated. ${ }^{4,9,22}$ The procedure was pioneered by Strong in 1958 when he stressed on the significance of mechanical obstruction on the duodenum caused by high insertion of the ligament of Treitz. It involves division of the ligament of Treitz with mobilization of the transverse and ascending duodenum caudally, away from the apex of the aortomesenteric angle. ${ }^{8}$ However, this technique was reported to have an unacceptably high failure rate, probably due to post-operative adhesions and the interference of downward duodenal displacement by the short branches of inferior pancreaticoduodenal artery to the duodenum. ${ }^{11}$ Hence, modification of this technique has been proposed and extensively practiced since 1974. Passage of the jejunum and ileum underneath the superior mesenteric vessels into the right side of the abdomen following Strong's procedure can give a better outcome. The ascending colon is then separated from its retroperitoneal attachments and placed in the left side of the abdomen. The end result is a configuration similar to that seen in congenital mal-rotation of the intestine. ${ }^{8}$ One of the respondents in our study first underwent Strong's procedure laparoscopically, but it was deemed unsuccessful. An open duodenal de-rotation was then suggested as the ligament of Treitz had been severed during the first procedure. Intraoperatively, the ligament was found adhered to its original place, suggesting post-operative adhesion formation. The patient reported that all of the pre-operative symptoms were almost completely alleviated. 
An overall review on the prognosis proved that majority of the patients are still in need of supervision regardless of their method of treatment. About $87 \%$ of our cases that had received treatment are still struggling with the ongoing symptoms that either persisted or re-appeared. Five patients (14.3\%) were diagnosed with SMAS and had yet to receive any interventions.

Some limitations of our study were clearly recognized. In an online survey, participation is usually unpredictable. Only a small number of cases enrolled in our study and majority of them were living in the same continent, rendering a difficult evaluation on the worldwide management trend. In addition, some patients might have provided inaccurate or inadequate information regarding their condition due to recall bias. An extensive future study with a large number of cases using a proper study design is advocated.

As a conclusion, diagnosis of SMAS is established after a thorough assessment of the clinical presentations and confirmation with suitable imaging modalities. The similarity of clinical presentations in SMAS and other gastrointestinal issues might complicate the diagnosis; however, they can be distinguished easily by certain imaging techniques which can identify the decrease in the aortomesenteric angle and distance. The choice of treatment should be dependent on the causes and severity, as different patients respond differently to various therapies. Recurrence of obstruction or development of complication is possible and thus a long-term follow up plan is required in all patients.

\section{References}

1. Ahmed AR, Taylor I. Superior mesenteric artery syndrome. Postgrad Med J. $1997 \operatorname{Dec} ; 73(866): 776-8$.

2. Akin JT Jr, Skandalakis JE, Gray SW. The anatomic basis of vascular compression of the duodenum. Surg Clin North Am. 1974 Dec;54(6):1361-70.

3. Alhadi AN, Shuqdar RM. Anorexia nervosa versus superior mesenteric artery syndrome in a young woman: case report and literature review. Journal of Taibah University Medical Sciences. 2008;3(1):55-60.

4. Delgadillo X, Belpaire-Dethiou MC, Chantrain C, Clapuyt P, Veyckemans $F$, de Ville de Coyet J, et al. Arteriomesenteric syndrome as a cause of duodenal obstruction in children with cerebral palsy. J Pediatr Surg. 1997 Dec;32(12):1721-3.

5. Lee TH, Lee JS, Jo Y, Park KS, Cheon JH, Kim YS, et al. Superior mesenteric artery syndrome: where do we stand today?. J Gastrointest Surg. 2012 Dec;16(12):2203-11.

6. Lippl F, Hannig C, Weiss W, Allescher HD, Classen M, Kurjak M. Superior mesenteric artery syndrome: diagnosis and treatment from the gastroenterologist's view. J Gastroenterol. 2002 Aug;37(8):640-3.

7. Biank V, Werlin S. Superior mesenteric artery syndrome in children: a $20-$ year experience. J Pediatr Gastroenterol Nutr. 2006 May;42(5):522-5.

8. Ha CD, Alvear DT, Leber DC. Duodenal derotation as an effective treatment of superior mesenteric artery syndrome: a thirty-three year experience. Am Surg. 2008 July;74(7):644-53.

9. Hines JR, Gore RM, Ballantyne $\mathrm{CH}$. Superior mesenteric artery syndrome. Diagnostic criteria and therapeutic approaches. Am J Surg. 1984 Nov; 148(5):630-2.

10. Jones PA, Wastell C. Superior mesenteric artery syndrome. Postgrad Med J. 1983 Jun 1;59(692):376-9.

11. Lorentziadis ML. Wilkie's syndrome. A rare cause of duodenal obstruction. Ann Castroenterol. 2011;24(1):59-61.

12. Raissi B, Taylor BM, Taves DH. Recurrent superior mesenteric artery (Wilkie's) syndrome: a case report. Can J Surg. 1996 Oct;39(5):410-6.

13. Wilkie DP. Chronic duodenal ileus. Am J Med Sci. 1927;173(5):643-8.

14. Mathenge N, Osiro S, Rodriguez II, Salib C, Tubbs RS, Loukas M. Superior mesenteric artery syndrome and its associated gastrointestinal implications. Clin Anat. 2014 Nov;27(8):1244-52.

15. Welsch T, Buchler MW, Kienle P. Recalling superior mesenteric artery syndrome. Dig Surg. 2007;24(3):149-56.

16. Neri S, Signorelli SS, Mondati E, Pulvirenti D, Campanile E, Di Pino L, et al. Ultrasound imaging in diagnosis of superior mesenteric artery syndrome. J Intern Med. 2005 Apr;257(4):346-51.

17. Zhu ZZ, Qiu Y. Superior mesenteric artery syndrome following scoliosis surgery: its risk indicators and treatment strategy. World J Gastroenterol. 2005 Jun 7;11(21):3307-10.

18. Barnes JB, Lee M. Superior mesenteric artery syndrome in an intravenous drug abuser after rapid weight loss. South Med J. 1996 Mar;89(3):331-4.

19. Merret ND, Wilson RB, Cosman P, Biankin AV. Superior mesenteric artery syndrome: diagnosis and treatment strategies. J Gastrointest Surg. 2009 Feb;13(2):287-92.

20. Geer DA. Superior mesenteric artery syndrome. Mil Med. 1990 Jul;155(7): 321-3.

21. Ylinen P, Kinnunen J, Hockerstedt K. Superior mesenteric artery syndrome. A follow-up study of 16 operated patients. J Clin Gastroenterol. 1989 Aug;11(4):386-91.

22. Bermas H, Fenoglio ME. Laparoscopic management of superior mesenteric artery syndrome. JSLS. 2003 Apr-Jun;7(2):151-3.

23. Barner $H B$, Sherman CD Jr. Vascular compression of the duodenum. Int Abstr Surg. 1963 Aug;117:103-18.

24. Burrington JD. Superior mesenteric artery syndrome in children. Am J Dis Child. 1976 Dec;130(12):1367-70.

25. Wilkie DP. Chronic Duodenal Ileus. Br J Surg. 1921;9(34):204-14.

\footnotetext{
Acknowledgments

We thank the members of the "Superior Mesenteric Artery (SMA) Syndrome Awareness \& Support" Facebook group for their cooperation and support in conducting this study.

Conflict of Interest Statement at Funding

The authors have no funding, financial relationships, or conflicts of interest to disclose.

Author Contributions

Conceptualization, Data analysis and interpretation, Critical revision of the manuscript, Approval of the final version: RA, HAR, DSA, MRE. Writing: RA, HAR, DSA. Statistical advice: MRE.

Cite as:

Awaludin R, Ab Rahim H, Arivai DS, Elkeleny MR. Superior mesenteric artery syndrome: a worldwide descriptive study with literature review. Int J Med Students. 2016 May-Aug;4(2):50-4.
} 